livraisons

d'Histoire

de l'Architecture

\section{Livraisons de l'histoire de l'architecture}

$33 \mid 2017$

Histoire du (des ?) patrimoine(s)

\title{
Le programme de transport supersonique concorde : de l'innovation à la patrimonialisation
}

"The supersonic transport Concorde: from innovation to heritage enhancement „Das Überschallflugzeug Concorde : von der technischen Innovation zum kulturellen Erbe“

David Berthout

\section{(2) OpenEdition}

\section{Journals}

Édition électronique

URL : http://journals.openedition.org/lha/746

DOI : 10.4000//ha.746

ISSN : 1960-5994

Éditeur

Association Livraisons d'histoire de l'architecture - LHA

Édition imprimée

Date de publication : 15 juin 2017

Pagination : 73-85

ISSN : $1627-4970$

\section{Référence électronique}

David Berthout, "Le programme de transport supersonique concorde : de l'innovation à la

patrimonialisation », Livraisons de l'histoire de l'architecture [En ligne], 33 | 2017, mis en ligne le 16 juin 2019, consulté le 16 octobre 2019. URL : http://journals.openedition.org//ha/746 ; DOI : 10.4000/lha. 746 


\section{LE PROGRAMME DE TRANSPORT SUPERSONIQUE CONDORDE : DE L'INNOVATION À LA PATRIMONIALISATION}

$\mathrm{Au}$ cours du $\mathrm{XX}^{\mathrm{e}}$ siècle, les progrès de l'aviation sont fulgurants. Moins de quarante-cinq ans séparent le premier vol homologué d'un "plus lourd que l'air ", réalisé en 1903 par les frères Wright ${ }^{1}$ et le premier vol supersonique effectué en 1947 par l'aviateur américain Chuck Yeager à bord du Bell X-1. À peine cinquante années séparent la première traversée aérienne de l'Atlantique entre New-York et Paris par Lindberg en mai $1927^{2}$ et les vols commerciaux réguliers entre l'Europe et les Amériques, effectués dès 1976 à une vitesse dépassant celle du son ${ }^{3}$.

À la suite du premier vol de Yeager en 1947 à bord d'un prototype, plusieurs avions militaires supersoniques sont produits en série : le F-100 Super Sabre américain à partir de 1954, le Mikoyan-Gourevitch Mig-19 Farmer soviétique à partir de 1955 et le Super Mystère B2 français à partir de 1958. Mais il faut encore attendre plusieurs années pour que cette technologie, dans un premier temps réservée aux avions de chasse, soit adaptée au transport civil.

L'histoire de l'avion de transport supersonique (TSS) Concorde a tout d'une épopée. Né de la volonté politique de deux pays, la France et la Grande-Bretagne et d'un investissement industriel du secteur privé, il est le fruit d'une extraordinaire prouesse technologique. Mais, à l'instar des héros déchus et en dépit de la fascination qu'il a toujours inspirée, il dut affronter tout au long de son exploitation de nombreuses difficultés et connut une fin tragique.

Et pourtant, Concorde était l'enfant prodige à qui tout était promis. Après vingt ans de recherche et d'essais, le supersonique s'impose très vite comme un

1. Certains estiment que c'est Clément Ader qui aurait effectué le premier vol d'un "plus lourd que l'air " en 1890 à bord de l'Éole I, mais cette affirmation reste toujours sujette à caution.

2. Sans oublier, quinze jours avant Lindberg, la tentative de Nungesser et Coli dans le sens Paris/ New-York, plus difficile car en vent contraire et qui s'est terminée tragiquement. Certains pensent aujourd'hui que les deux aviateurs ont réussi leur traversée et se sont abîmés en mer tout près de Saint Pierre-et-Miquelon dans des circonstances suspectes. Selon une version, ils auraient été pris pour cible par des contrebandiers Saint-Pierre-et-Miquelonais trafiquant de l'alcool avec les ÉtatsUnis (en pleine prohibition), lesquels auraient tiré sur l'Oiseau Blanc, dans le brouillard, en le prenant pour un avion de gardes côtes américains.

3. La vitesse supersonique se mesure en Mach, en l'honneur du philosophe et physicien autrichien Ernst Mach. La vitesse Mach est une convention qui n'est pas fondée sur une valeur fixe. Elle est en effet calculée à partir de la vitesse du son, laquelle varie en fonction de l'environnement dans lequel le son circule. On parle de vitesse subsonique quand elle est inférieure à Mach 1, de vitesse transsonique quand elle est de Mach 1, de vitesse supersonique quand elle dépasse Mach 1 et de vitesse hypersonique quand elle dépasse Mach 5. 
avion majeur, une "cathédrale aéronautique " témoignant du savoir-faire des ingénieurs du $\mathrm{XX}^{\mathrm{e}}$ siècle. Or, en dépit des réussites technologique et humaine indéniables, le constat d'échec commercial est inévitable.

\section{Conception et développement}

\section{Préfiguration}

Dès le milieu des années 1950, la France et la Grande-Bretagne étudient, chacun de leur côté, la possibilité de réaliser un avion supersonique conçu pour le transport des passagers. L'administration française envisage officiellement cette possibilité en 1956 et la même année, le gouvernement anglais crée un comité d'étude regroupant tous les secteurs publics concernés par l'aéronautique. Ce comité est chargé d'évaluer la faisabilité du projet et de lancer des recherches sur l'aérodynamique et la structure à donner au futur avion supersonique.

En 1957-1958, les secteurs privés de l'industrie aéronautique s'intéressent à leur tour au projet, dont le français Sud Aviation qui étudie le moyen de développer le Super Caravelle. En 1959, le ministère de l'Air charge officiellement Sud Aviation, Nord Aviation et Marcel Dassault de concevoir un avant-projet d'avion moyen courrier supersonique.

En 1959, côté britannique, le comité d'étude se dirige vers un long courrier pouvant atteindre Mach 2,2. L'année suivante, le gouvernement anglais charge British Aircraft Corporation (BAC), tout juste créée ${ }^{4}$, de mettre au point un avant-projet détaillé, lequel doit s'appuyer sur les travaux déjà effectués par Bristol Aeroplane Company sur le Bristol 223.

Au tournant de 1960, les entreprises aéronautiques et les administrations des deux pays continuent de travailler indépendamment. Français et Britanniques souhaitent en effet contrer l'hégémonie américaine. Sud Aviation et Dassault mettent en commun leur expérience sur, respectivement, Super Caravelle et les Mirage III et IV, Durandal et Trident. Cela leur permet de proposer en mai 1961 au gouvernement un avant-projet de Super Caravelle prévue pour le transport de 76 ou 84 passagers, projet retenu en octobre suivant par les services techniques du ministère de l'Air. Entre temps, au mois de juin 1961, une maquette de l'avion quadrimoteur est présentée au Salon du Bourget, tandis que le stand de l'anglais BAC présente une maquette de son avion à 6 moteurs.

Cependant, les coûts importants de développement incitent Français et Britanniques à envisager une collaboration. Côté officiel, les premières rencontres ont lieu en juin et juillet 1961. De fin 1961 à octobre 1962, les deux pays discutent de l'accord qu'ils souhaitent mettre en place et qu'ils signent le 29 novembre 1962.

4. En janvier 1960, British Aircraft Corporation (BAC) est créée par la fusion de Bristol Aeroplane Company, English Electric, Vickers-Armstrong et, en septembre de la même année, de Hunting Aircraft. 
Côté industriels, BAC et Sud Aviation s'étaient déjà rapprochés dès le printemps 1960 et avaient échangé sur leurs projets respectifs. Les motoristes français SNECMA et anglais Bristol Siddeley Engines Limited (BESL) signent quant à eux un accord en novembre 1961 pour concevoir, construire et proposer conjointement des moteurs d'avion supersonique. En décembre 1961, Sud Aviation et BAC s'entendent sur les caractéristiques générales du futur avion. Seul le rayon d'action demeure un point de désaccord entre les deux industriels, Sud Aviation restant sur un moyen courrier, BAC sur un long courrier pour les vols transatlantiques.

Concrètement, l'accord franco-britannique signé en novembre 1962 pose le principe de collaboration sur la base d'un partage égal des responsabilités, des dépenses, mais aussi des fruits de l'exploitation commerciale à venir, entre les administrations et les entreprises des deux pays. L'accord table sur un engagement de dépenses évalué à 1865 millions de nouveaux francs étalés entre 1962 et 1969. Techniquement, l'accord prévoit deux versions, moyen et long courrier, réalisées par deux industries de chaque pays : Sud Aviation/BAC pour les cellules et SNECMA/BESL pour les moteurs ${ }^{5}$, regroupées dans une organisation créée pour l'occasion. Enfin, un Comité permanent composé de fonctionnaires des deux pays doit superviser le programme.

Le programme d'avion supersonique désormais franco-anglais est donc tout à la fois une entreprise industrielle privée et la volonté politique de deux pays qui inscrivent leur action dans un cadre de prestige national. Cette volonté paraît d'autant plus importante que la France comme le Royaume-Uni, bien que sortis "vainqueurs " du Second conflit mondial, membres du Conseil de sécurité permanent de l'ONU et parmi les principaux pays industrialisés du monde, sentent leur influence décliner. Entre les mouvements de décolonisation entamés dès la fin de la guerre et qui s'accélèrent, mettant à mal leur statut de puissance planétaire, et la primauté américano-soviétique, la conception et la fabrication d'un avion tel que le supersonique est pensé comme l'affirmation de la grandeur technologique et industrielle du "vieux continent». Et l'orgueil national se niche parfois dans les détails, même entre partenaires. Ainsi, quand il s'agit de baptiser le futur avion, une polémique s'installe. Non pas sur le nom, qui fait consensus, mais sur son orthographe, les Anglais souhaitant un Concord sans «e" final, les Français, bien sûr, exigeant une orthographe francophone, et donc un «e" final.

\section{Essais}

Après la phase de conception qui prend plusieurs années et qui continue même après le début des essais, vient la phase d'expérimentation, qui va occuper plus de 10 ans les équipes du programme. Toutes les pièces de l'appareil sont testées et retestées, séparément ou ensemble. En mars 1963, décision est prise que le premier prototype sera assemblé à Toulouse. Au même moment, le ministre britannique de

5. Le moteur est un Bristol Olympus. BESL sera racheté par Rolls Royce en 1968. La SNECMA appartient aujourd'hui au groupe SAFRAN et équipe les Airbus, Boeing et les fusées Ariane. 
la Technologie Tony Benn annonce que Concorde sera écrit avec un "e " final, lettre que l'on retrouve, selon ses propres mots, dans Excellence, England, Europe et Entente, se référant ainsi à l'Entente Cordiale liant les deux pays.

En mai 1963, la date du premier vol du prototype Concorde 001 (Sud Aviation) est fixée au mois de juin 1966 et au mois de décembre 1966 pour le prototype Concorde 002 (BAC). Les délais et le planning évoluent bien évidemment au fil de la décennie, variant en fonction des aléas techniques. Ainsi, le premier vol du Concorde 001 est très rapidement repoussé au premier trimestre 1967, puis à novembre 1967, puis au premier trimestre 1968, pour finalement décoller pour la première fois le 2 mars 1969 de Toulouse-Blagnac et réaliser un vol de 42 minutes. Le pilote d'essai de ce premier vol est André Turcat ${ }^{6}$, figure devenue mythique du milieu aéronautique français. Le premier vol du Concorde britannique 002 a lieu quant à lui le 9 avril 1969, piloté par Brian Trubshaw. Le mur du son est passé la première fois par Concorde 001 le $1^{\text {er }}$ octobre 1969 et par Concorde 002 le 25 mars 1970. Parallèlement, en 1969, les autorités donnent le feu-vert pour la construction, en plus des deux prototypes 001 et 002 , de deux avions de pré-série, de deux avions pour les essais au sol et de trois avions de série.

\section{Innovations et caractéristiques techniques}

Au final, les avions de série, construits à Toulouse pour la France et à Filton pour la Grande-Bretagne, mesureront 62,17 mètres de longueur, auront une envergure de 25,60 mètres pour un poids de 185 tonnes de charge maximale au décollage et une capacité de 128 passagers plus l'équipage. Outre sa vitesse, qui est sa caractéristique la plus connue et la plus impressionnante pour le grand public, de nombreuses innovations techniques caractérisent Concorde. Or ces innovations sont à la base de la technologie de nombreux avions de ligne actuels, notamment en matière électronique, principalement dans la série des Airbus. En effet, les deux propriétaires, BAC et Aérospatiale donnent naissance respectivement à BAE Systems et EADS, regroupés ensuite au sein d'Airbus.

La vitesse de croisière de Concorde est de Mach 2,02 pour une altitude moyenne située entre 16000 et 18000 mètres. L'avion est équipé d'ailes delta spécifiques dites gothiques. Concorde est le premier avion civil équipé de commandes entièrement électriques et analogiques et d'un pilotage automatique. Les circuits hydrauliques et l'électricité sont gérés par IDG (Integrated Driving Generator) et le freinage est doté d'un système perfectionné antidérapant (SPAD) qui permet de réduire les distances d'arrêt de $15 \%$. Ce système viendra par la suite équiper les avions Airbus et les aéronefs militaires français. L'avion comporte aussi un système permettant le rééquilibrage des masses pour une optimisation des performances, notamment par le déplacement du carburant afin de positionner au mieux le centre de gravité en cours de vol. Les pièces qui le structurent sont usinées d'un seul tenant et ne sont

6. André Turcat (1921-2016), aviateur, pilote dans l'armée, pilote d'essais chez Nord Aviation puis chez Sud Aviation sur le Concorde. 
pas composées par l'assemblage de petites pièces, ce qui permet de réduire le nombre et la masse des composants. Enfin, certaines de ces pièces sont en matériaux composites.

Ces innovations rendent le coût de fabrication de Concorde supérieur à celui d'un avion classique, mais elles sont aussi un investissement. En effet, nombre d'entre elles ont 20 ans d'avance au moment de leur conception, permettent de continuer le programme et deviennent par la suite des équipements standards dans l'aéronautique civile. Par ailleurs, cette technologie de pointe a été constamment renouvelée et améliorée au cours du programme. Les concepteurs de l'avion ne se sont en effet pas reposés sur leurs lauriers et ont envisagé dès le début de l'exploitation commerciale une nouvelle version afin de réduire le bruit de l'avion et de porter sa capacité de distance de 6800 à $7500 \mathrm{~km}$. Ces travaux de recherche et de conception ont entraîné des modifications sur l'aérodynamisme en augmentant l'envergure pour accroître la finesse de l'avion, ainsi que sur les moteurs, pour renforcer la poussée et réduire la consommation de carburant et le bruit.

\section{Concurrence}

En dehors de la France et de la Grande-Bretagne, deux autres pays se lancent dans la conception d'un avion de transport supersonique. Tout d'abord les ÉtatsUnis, où deux programmes concurrents voient le jour. Le Lockheed L-2000, assez similaire au Concorde, et le Boeing 2707, plus audacieux. Ce dernier, en titane, possède une voilure à géométrie variable et compte faire voyager 300 passagers à Mach 2,7, donc plus rapidement que Concorde. C'est ce modèle qui est choisi dans un premier temps par le Congrès américain. Mais le projet, moins avancé que le programme européen, est confronté à une vive opposition pour des raisons, en partie, environnementales. Finalement, après un refus du Sénat et de la Chambre des représentants de lui accorder les crédits nécessaires, il est abandonné en mars 1971.

Le seul avion concurrent non seulement conçu, mais aussi usiné et mis en service, est le Tupolev TU-144 soviétique. Cet avion doit une partie de sa conception à l'espionnage industriel, notamment des usines de Sud Aviation. La guerre froide et la compétition entre l'URSS et les puissances occidentales se jouent sur les plans politique et militaire, mais aussi technologique, industriel et commercial. Certains épisodes sont d'ailleurs dignes d'un scenario de film d'espionnage et soulignent à merveille la guérilla menée entre l'Est et l'Ouest. En 1965, Sergei Pavlov, directeur du bureau parisien de la compagnie Aeroflot, est arrêté à Paris avec sur lui les plans des freins, du train d'atterrissage et de la cellule de Concorde. 12 ans plus tard, en 1977, c'est Sergei Fabiew, directeur de la Société générale d'études et de réalisations industrielles (SERGI) qui est arrêté. À cette époque, la SERGI a comme client, entre autres, l'avionneur Dassault. Or Fabiew était à la tête d'un réseau d'espionnage qui avait à son actif, parmi d'autres forfaits, le vol des plans de modèles de voitures conçues par Fiat en Italie.

Le Tupolev TU-144 vole pour la première fois le 31 décembre 1968 pendant 38 minutes et passe le mur du son le 5 juin 1969. Le 3 juin 1973, lors d'un vol 
de démonstration au Salon du Bourget, un TU-144 s'écrase au sol à Goussainville, tuant les 6 membres d'équipage, 8 personnes au sol, détruisant 15 maisons et une école, heureusement fermée ce jour-là. Cet accident entraîne un retard dans le développement de l'avion qui entre finalement en service le 6 décembre 1975 au sein de la compagnie aérienne russe Aeroflot, mais uniquement pour le fret et le courrier. La première ligne d'exploitation commerciale sur des vols passagers ouvre en novembre 1977 sur une ligne reliant Moscou à Alma-Ata au Kazakhstan. Le TU-144 peut voler à plus de Mach 2 et transporter de 98 à 120 passagers à une altitude de 20000 mètres. Son rayon d'action s'étend jusqu'à 6200 kilomètres. Cependant, mal réalisé, le TU-144 connaît plusieurs problèmes techniques et un nouvel accident se produit le 23 mai 1978 provoquant le crash de l'avion, la mort de 2 membres de l'équipage et sonnant le glas des vols réguliers pour passagers. Le TSS russe continue pourtant par la suite à voler jusqu'en 1983, date de son retrait du service, mais uniquement comme avion-cargo. En définitive, le Tupolev TU144, avion relativement peu fiable, peu utilisé en dehors du fret et seulement sur Aéroflot, n'aura pas été un véritable concurrent commercial pour Concorde.

\section{Exploitation commerciale}

L'exploitation commerciale de Concorde n'a pas été une partie de plaisir. Dès le départ, les difficultés se sont accumulées: lignes régulières difficiles à installer, commandes d'avions de compagnies aériennes annulées, choc pétrolier, procès retentissant aux États-Unis... Et pourtant, Concorde a volé pendant 27 ans.

\section{Vols réguliers}

Les avionneurs français et britanniques qui ont mis au point et construit Concorde espèrent évidemment le vendre aux compagnies aériennes du monde entier. C'est pourquoi de gros efforts de communication et de marketing sont déployés alors même que l'avion est toujours en phase d'assemblage et d'essais : inauguration par la reine d'Angleterre du centre d'essai au Royaume-Uni en 1966, présentation de la maquette aux compagnies aériennes internationales et présentation de la maquette en grandeur nature au salon du Bourget de 1967, vols de démonstration en 1971, tournées de présentation en Amériques du Nord et du Sud, au Moyen-Orient, en Extrême-Orient et en Australie au début des années 1970, vols organisés pour les autorités politiques et industrielles internationales. Parallèlement, les récompenses pleuvent : la Fédération Aéronautique Internationale délivre un diplôme d'honneur à l'équipe franco-britannique à l'origine de Concorde et les deux pilotes d'essais André Turcat et Brian Trubshaw sont honorés d'un trophée décerné par l'Association des Pilotes d'Essais des États-Unis.

La stratégie commerciale semble tout d'abord porter ses fruits. En avril 1967, 16 compagnies aériennes, dont 8 nord-américaines, ont mis une option d'achat portant sur un total de 74 Concorde. En 1972, des contrats préliminaires sont encore signés pour la vente de Concorde avec des compagnies en République populaire 
de Chine, en Iran et en France. Mais, pour des raisons de modifications et d'adaptations techniques, la date de mise en service de Concorde originellement prévue en 1971 a été plusieurs fois reportée, pour être finalement fixée à janvier 1976. Or, entre 1956, début des études, 1962, date de l'accord franco-britannique, et 1976, début de l'exploitation commerciale, la conjecture a sensiblement changé.

Les hypothèses économiques et écologiques sur lesquelles le programme Concorde a été bâti ont en effet évolué. De gros porteurs subsoniques de deuxième génération entrent en service à la fin des années 1960, moins bruyants, offrant une capacité de transport en nombre de passagers plus importante que leurs prédécesseurs ainsi qu'une moindre consommation en carburant. L'économie mondiale entre dans une période de stagnation et d'inflation mettant les compagnies aériennes en défaut d'investir dans des supersoniques alors qu'elles ont déjà beaucoup dépensé pour l'achat des subsoniques de deuxième génération. Le choc pétrolier, fin 1973, rend le combustible beaucoup plus cher. Par ailleurs, des règles internationales adoptées en 1969 fixent les niveaux de bruit autour des aéroports. Enfin et en conséquence, beaucoup de pays interdisent les vols supersoniques au-dessus de leur territoire.

Or, les adaptations et modifications apportées sur Concorde pour faire face à ces problèmes, celles-là mêmes qui avaient en partie provoqué le retard de mise en service, ne sont pas suffisantes pour convaincre les acheteurs potentiels. En janvier 1973, la compagnie américaine Pan-Am revient sur ses commandes, sans remettre en cause pour autant les aptitudes de l'avion. Elle est immédiatement suivie par la TWA qui annule à son tour ses réservations. C'est une condamnation commerciale du programme Concorde. Au final, sur la soixante-dizaine de pré-commandes, seules celles d'Air France et de British Airways, sous la pression des gouvernements français et britannique, sont transformées en achats fermes.

C'est dans ces conditions que l'exploitation commerciale de Concorde sur des lignes régulières de passagers démarre le 21 janvier 1976. Les premiers trajets assurés par Air France et British Airways sont Paris-Rio de Janeiro, Paris-Caracas et Londres-Barhein. Jusqu'en 1983, les destinations des Concorde d'Air France sont Rio de Janeiro, Caracas, Dakar, Mexico, Washington, Dallas et New-York, dont la desserte n'aura pas été sans poser de grosses difficultés. À partir de 1983 et jusqu'en 2003, pour des raisons de rentabilité, Air France supprime toutes ses destinations sauf New-York, et met en place un certain nombre de vols affrétés sur demande ou en fonction d'événements particuliers. Seule exception, en septembre 1995, la Chine accorde l'autorisation à Air France et British Airways de faire atterrir leurs Concorde à Pékin. Mais le bruit au décollage amène les autorités chinoises à interdire Pékin au Concorde. Dès lors, les escales se font à Tianjin, en bord de mer, à $140 \mathrm{~km}$ de Pékin.

Outre Air France et British Airways et en association avec cette dernière, l'avion vole brièvement en 1977 sous les couleurs de Singapore Airlines sur la ligne ChangiBarhein, expérience abandonnée au bout de quelques mois pour des raisons de nuisances sonores. Nouvelle tentative en 1979, là aussi avortée, pour les mêmes raisons. À la même époque, une compagnie aérienne loue deux Concorde pour une 
exploitation subsonique au-dessus du sol américain et supersonique entre les USA et l'Europe. Mais cette exploitation n'étant pas bénéficiaire, la compagnie y met un terme en 1980.

\section{L'opposition à Concorde: les problèmes aux États-Unis}

Dans le même temps, un sentiment de rejet prend naissance et se développe aux États-Unis, sentiment qui s'exprime très concrètement par des recours judiciaires contre Concorde. Une des explications souvent retenue pour expliquer ce rejet trouve son fondement dans la croyance d'une volonté américaine de couler un fleuron de l'industrie aéronautique européenne, concurrent sérieux des avionneurs et des compagnies aériennes US.

Les faits sont évidemment un peu plus compliqués. Tout d'abord, l'argument consistant à affirmer que Concorde a fait l'objet d'un véritable sabotage commercial pour ne pas concurrencer l'industrie aéronautique made in USA trouve sa limite dans l'abandon dès 1971 du TSS américain : il n’y a pas de concurrent américain, du moins direct, à Concorde ${ }^{7}$. Par ailleurs, avant d'être abandonné, le projet américain de TSS avait lui-même été exposé à de nombreuses critiques en interne, principalement liées à des considérations écologiques et de nuisances sonores, critiques qui se sont par la suite naturellement reportées sur Concorde. L'opposition au transport sonique s'inscrit par ailleurs dans un mouvement plus général. En 1969 est votée l'"Environmental Protection Act ", loi fédérale instituant l'obligation de procéder à une enquête d'utilité publique pour évaluer l'impact écologique de toute nouvelle entreprise. Or c'est cette loi qui a permis ensuite, ses opposants de s'opposer à l'atterrissage de Concorde aux États-Unis en portant la lutte sur les plans administratif, légal et judiciaire.

Administrativement, c'est la Federal Aviation Authority (FAA ${ }^{8}$ qui a été chargée de diligenter l'enquête. La publication d'un projet de déclaration d'impact sur l'environnement ${ }^{9}$ est parue le 3 mars 1975 et a été suivie de plusieurs audiences et remises de rapports, à l'issue desquelles le ministre américain des transports a autorisé le 4 février 1976 Air France et British Airways à faire atterrir leurs Concorde à New-York et Washington pendant une période d'essai de 16 mois. L'état fédéral américain ne s'est donc pas montré hostile au TSS franco-anglais. L'aéroport Dulles de Washington, propriété gouvernementale, s'est d'ailleurs plié à la décision.

Ce n'est pas le cas de l'aéroport de New-York, propriété conjointe des états de New-York et du New-Jersey et géré par la Port of New-York Authority (PONYA) ${ }^{10}$,

7. Ce point serait sûrement à approfondir. La technologie de pointe mise en place sur Concorde pouvait inquiéter les constructeurs aéronautiques américains si jamais elle venait à être utilisée par les constructeurs européens sur d'autres avions de ligne. Ce qui a été le cas puisqu'elle est en partie à l'origine de la technologie utilisée pour la construction des Airbus et de la réussite de ce constructeur. Mais, au début des années 1970, il était difficile de prévoir quel serait l'apport de Concorde à l'aéronautique européenne.

8. Il s'agit de l'équivalent américain de la direction générale de l'Aviation civile (DGAC) française.

9. «Draft Environmental Impact Statement".

10. Aujourd'hui Port Authority of New-York and New-Jersey. 
laquelle a su mettre à profit le droit américain qui prévoit que toute personne privée ou morale peut recourir contre une décision administrative si elle s'estime lésée dans ses intérêts. Le 11 mars 1976, la PONYA interdit donc l'atterrissage de Concorde à New-York pour une durée minimale de six mois en raison de l'impact du bruit de l'avion sur les riverains. En réaction, Air France et British Airways déposent une plainte devant la cour du district sud de l'état de New-York, arguant d'inconstitutionnalité de la décision contrevenant à une décision fédérale. Après plus d'un an de procédure, la justice américaine décide que l'interdiction à l'encontre de Concorde est «discriminatoire, arbitraire et déraisonnable ". C'est donc une victoire pour les compagnies française et britannique qui rouvrent la desserte de New-York le 28 novembre 1977.

Parallèlement aux procédures administrative et judiciaire, des actions législatives sont lancées. Du côté des " anti ", des propositions pour interdire le ciel américain à Concorde sont proposées à certaines commissions parlementaires du Sénat et de la Chambre des représentants. Ces oppositions ne sont cependant pas spécifiquement anti Concorde mais plus généralement anti supersonique puisque, parmi les opposants les plus acharnés, se trouve le député de New-York Lester Wolff, qui avait déjà été à la tête de la fronde anti supersonique "made in USA".

Côté "pro", des actions de lobbying sont menées. En 1975, l'Aérospatiale signe un contrat avec un consultant américain chargé de porter la bonne parole auprès des autorités fédérales et locales ainsi qu'auprès des médias. Le travail dut être efficace, car les projets de loi hostiles aux supersoniques n'aboutirent pas. En septembre 1977, le secrétaire d'État aux transports Brock-Adams propose une solution intermédiaire autorisant l'atterrissage de Concorde dans les aéroports de 13 grandes villes américaines, dont New-York. Ce règlement prévoit aussi que les 16 Concorde produits avant 1980 ne soient pas soumis à la réglementation sur le bruit, contrairement aux supersoniques à venir. Il limite par ailleurs les plages horaires d'atterrissage de 7 heures à 22 heures et interdit de produire un "bang " sonique - et donc de franchir le "mur du son", si le bruit peut être entendu sur le territoire des USA.

Finalement, même si le résultat des actions menées de part et d'autre est relativement en faveur de Concorde, la publicité négative faite principalement autour du bruit - réel ou exagéré - produit par l'avion nuit grandement à son image et incite d'autres pays à promulguer des règlements réduisant l'accès de Concorde à leurs aéroports. La situation est telle qu'Air France et British Airways doivent faire évoluer leur offre en matière de vols commerciaux supersoniques.

\section{Vols spéciaux}

Afin de rentabiliser les appareils qui coûtent cher en entretien et équipages et de palier les difficultés rencontrées sur les lignes aériennes pérennes, Air France et British Airways décident de créer des vols charters, c'est-à-dire des vols programmés à la demande pour des entreprises ou des agences de voyage. Des vols spécifiques liés à des occasions particulières sont aussi mis sur pied. Ces formules accom- 
pagnent des événements comme le transport de la flamme olympique pour les jeux d'hiver d'Albertville en 1992, la coupe du monde de football, le carnaval de Rio, le grand-prix de Formule 1, des meetings d'aviation ou encore en complément de croisières.

Les deux compagnies organisent aussi des tours du monde. Ces voyages durent environ un mois et concernent surtout des passagers américains. Il y a, certaines années, jusqu'à 6 tours du monde chez Air France. Il arrive qu'à certaines périodes l'actualité politique vienne contrarier cette programmation : la première guerre du Golfe en 1991, la vague d'attentats en France en 1995 ou encore, la même année, la reprise des essais nucléaires français dans le Pacifique, très mal vus à l'étranger.

Une autre des spécificités de Concorde est le transport d'autorités, principalement des chefs d'état. Le 7 mai 1971, le président Georges Pompidou utilise un prototype de Concorde pour effectuer un voyage d'État. Il en profite pour donner une interview dans laquelle il fait la promotion du Concorde et de la technologie française et européenne. Entre 1981 et 1995, tous les voyages présidentiels français vers la Chine sont effectués en Concorde. L'avion est alors équipé de bureaux, de chambres et d'un système de chiffrement des communications. Outre les chefs d'état français, d'autres présidents et monarques ont utilisé le Concorde pour des vols réguliers à l'ONU dont le siège est à New-York, ou pour des vols vers l'Afrique, comme Mobutu Sese Seko au Zä̈re et Félix Houphouët-Boigny en Côte d'Ivoire. Le Pape Jean-Paul II a aussi utilisé le Concorde. Dans ce cas, la règle voulant que les pays recevant le Pape organisent son voyage vers sa destination suivante, la France a décidé de le faire voyager en Concorde lors de son départ après une visite en France en 1989.

\section{Maintenance et coûts}

Les vols intensifs en exploitation commerciale d'un supersonique étant une première, les conditions techniques de maintenance ont été plus complexes que pour un avion subsonique. Le haut niveau de technologie de l'avion et les contraintes de sécurité ont en effet nécessité un entretien adapté, avec un surcoût important en pièces et en main-d'œuvre. À titre de comparaison, la maintenance d'un Concorde est de 18 à 20 heures par heure de vol, contre 2 heures pour un avion subsonique. Par ailleurs, le nombre relativement réduit d'heures de vol entraîne une station au sol prolongée et donc des problématiques spécifiques de maintenance. Autre particularité de Concorde, l'apparition de l'électronique dans tous les circuits en commande et en surveillance, entraînant une redéfinition des métiers de mécanicien et d'électricien. Face à ces dilemmes, Air France et British Airways ont eu deux approches différentes. La compagnie britannique a créé un service de maintenance dédié au supersonique, tandis la française a opté pour une mutualisation des services d'entretien entre Concorde et certains Airbus, permettant ainsi d'occuper les mécaniciens à plein temps et de maintenir les compétences technologiques. Air France a aussi décidé d'affecter des mécaniciens à domicile sur les escales permanentes telles que New-York, de les envoyer ponctuellement sur les escales non pérennes et de les embarquer sur les tours du monde. 
Ainsi, cette maintenance de haut niveau a permis de régler les problèmes techniques rencontrés par Concorde. Ces complications "à l'usure ", inhérentes à tout nouvel aéronef, subsonique comme supersonique et difficilement prévisibles, apparaissent lors de la mise en service et de l'exploitation commerciale. En dépit des vols d'essai qui, pour Concorde, ont totalisé 5500 heures, soit proportionnellement quatre fois plus qu'un avion classique, ces problèmes touchèrent aux moteurs de l'avion, à sa structure et à certain systèmes. Ils furent traités au fur et à mesure de leur apparition et demeurèrent sans conséquence pour la sécurité des passagers.

\section{La fin de Concorde : de l'exploitation à la patrimonialisation}

Pendant toute la durée de son exploitation, Concorde représente un poids de plus en plus difficile à supporter. Sa forte consommation en carburant obère sa rentabilité dans un contexte de prix du pétrole tendu. En dehors des deux compagnies aériennes française et britannique, aucune autre compagnie n'achète l'avion qui n'est produit, au total, qu'à 20 exemplaires dont seulement 14 sont des avions commerciaux. En raison de l'opposition des états à l'accès du TSS à leurs aéroports, les lignes régulières commerciales sont confinées aux liaisons transatlantiques. Et même s'il demeure un avion emblématique et une figure de proue pour Air France et British Airways, les compagnies rechignent de plus en plus à faire voler ces avions dont l'achat même, à l'origine, avait été une décision politique imposée par les gouvernements plus qu'un choix stratégique commercial.

\section{L'accident de Gonesse}

C'est dans ce contexte que survient en 2000 l'accident dramatique de Gonesse. Ce 25 juillet, le vol 4590 d'Air France décolle de l'aéroport Roissy-Charles de Gaulle avec à son bord 100 passagers allemands et 9 membres d'équipage. Deux minutes plus tard, l'avion s'écrase sur un hôtel, tuant les 109 personnes à son bord et 4 personnes au sol. Cet accident est le seul mortel de l'histoire de Concorde. Après le crash, l'enquête est confiée au Bureau d'Enquêtes et d'Analyses pour la sécurité de l'aviation civile (BEA). Le rapport qu'il produit à la fin de ses investigations pointe du doigt la présence sur la piste d'une lame métallique perdue par l'avion ayant décollé juste avant Concorde, un DC-10 de la Continental Airlines. La lame métallique a provoqué l'éclatement d'un pneu de l'avion au moment où il roulait dessus. Un morceau du pneu est venu percuter une des ailes, remplie de carburant, provoquant une inflammation à l'origine de la perte de puissance de deux des moteurs sur les quatre, entraînant la chute de l'appareil.

À la suite de cet accident et du rapport du BEA, des modifications ont été apportées pour renforcer les conditions de sécurité des Concorde : amélioration des contrôles techniques, protections en kevlar des réservoirs de carburant, pneus plus résistants mis au point et fournis par Michelin. Mais en raison de ces modifications, le nombre de passagers est réduit d'une dizaine de places, rendant l'exploitation encore moins rentable. Par ailleurs, l'accident marque durablement les consciences 
et ébranle sérieusement la confiance dans la technologie de l'avion et sa sûreté, qui étaient un de ses grands points forts. Néanmoins, les deux lignes sont rouvertes le 7 novembre 2001, après plus d'un an de mise en veille.

\section{Retrait du service}

Après sa remise en service, l'avion connaît une nouvelle suite de difficultés techniques. Le 15 juillet et les 3 et 5 novembre 2002, des problèmes de moteur apparaissent. Le 27 du même mois c'est un problème majeur de gouverne d'un avion de British Airways qui inquiète. Finalement, après ce qui ressemble de plus en plus à une longue agonie, Air France et British Airways annoncent conjointement le 10 avril 2003 la fin des lignes Concorde pour l'année suivante. Parmi les raisons qui expliquent cet abandon sont avancés la baisse de fréquentation depuis l'accident de Gonesse, les coûts de maintenance devenus exorbitants, la crise du trafic aérien après les attentats du 11 septembre 2001, les nouvelles normes sur le bruit et la pollution ainsi que la hausse du prix du kérosène. Mais, derrière les raisons officiellement données et qui ont objectivement pesé dans la balance, figure aussi un autre événement d'importance pour les deux compagnies aériennes, l'annonce par le constructeur EADS de sa décision de ne plus assurer la maintenance des Concorde à partir d'octobre 2003.

\section{Concorde au musée}

Dès lors que la décision de l'arrêt de l'exploitation est prise, commencent les tournées d'adieux. Les derniers vols commerciaux Paris-New-York pour Air France ont lieu le 31 mai 2003. Le dernier vol non commercial, pour VIP, a lieu quant à lui le 3 juin 2003, aussi entre Paris et New-York. Une semaine de démonstration est organisée du 20 au 24 octobre 2003 et le 20 octobre, la reine Elisabeth II fait allumer le château de Windsor lors du passage de l'avion, honneur suprême réservé ordinairement aux avions de chefs d'État.

Les derniers vols de l'avion sont assez symboliques puisqu'ils sont destinés au convoiement de certains d'entre eux dans des institutions patrimoniales. Entre le 12 et le 27 juin 2003, plusieurs Concorde quittent Paris pour rejoindre des collections de musées aux États-Unis, en Allemagne et en France. Aujourd'hui, sur les 20 Concorde fabriqués, 18 sont conservés dans des musées en France (Musée de l'Air et de l'Espace du Bourget, Musée Aeroscopia de Toulouse ou Musée Delta Athis Paray Aviation), en Grande-Bretagne (National Museum of Flight, Imperial War Museum, Fleet Air Arm Museum, Brookland Museum, Airbus and the Bristol Aero Collection), en Allemagne (Auto \& Technik Museum Sinsheim) et aux États-Unis (National Air and Space Museum-Smithsonian Institution, Seattle's Museum of Flight, Intrepid Sea and Air Museum), ou exposés sur des aérodromes ou des aéroports français et britanniques et même sur l'aéroport de Grantley à la Barbade.

L'histoire de Concorde est aujourd'hui devenue un mythe. L'avion reste au cœur de ceux qui ont vécu l'aventure et des innombrables passionnés d'aéronautique. Tous, ingénieurs, mécaniciens, pilotes, personnels navigants, personnels au 
sol ou "simples amateurs", alimentent le rêve, soit en témoignant pour ceux qui participé au programme, soit en effectuant des recherches pour ceux qui l'ont rêvé. Mais si beaucoup s'attachent à l'objet Concorde, peu nombreux sont ceux qui s'intéressent à son histoire par le biais des archives.

Et pourtant les sources sont riches: aux Archives nationales avec les fonds de la direction générale de l'Aviation civile (DGAC), les fonds d'Air France ou encore les fonds privés donnés par l'Association des Professionnels de Concorde et du Supersonique (APCOS). Aux Archives départementales de la Haute-Garonne, riches de nombreuses archives des industries de l'aéronautique installées dans le toulousain et d'archives privées telles que celles du commandant Turcat, premier pilote d'essai français de Concorde. Et à l'étranger, les Archives nationales britanniques qui conservent le pendant anglais du programme. Sans oublier bien sûr les archives privées des industries de construction aéronautique.

Ces archives papiers, iconographiques, sonores et vidéo témoignent de près de cinquante ans d'une épopée scientifique, technique, politique, diplomatique, judiciaire et, bien sûr, humaine. Elles inscrivent Concorde dans la lignée des "grands " de l'aéronautique, de ceux qui cumulent les superlatifs : le plus beau, le plus innovant, le plus rapide, le plus performant... Et si, comme nous le soulignions en introduction, l'histoire de Concorde présente toutes les caractéristiques d'une tragédie au sens théâtral comme au sens propre, elle en distille aussi ce ressort littéraire qui dissimule dans une fin tragique les graines d'un avenir meilleur. Concorde a en effet transmis un héritage. La technologie conçue et développée pour l'avion a dans bien des cas posé la base d'un équipement standard de l'aéronautique mondiale. Elle est par la suite été utilisée directement par Airbus, l'un des deux premiers constructeurs aéronautiques au monde, si ce n'est le premier, pour équiper sa gamme d'avions et lui assurer une réelle réussite technologique et cette fois-ci, commerciale.

David BERTHOUT chef de la Mission Archives de la direction générale de l'Aviation civile 
